\title{
Prevalence of Manual Strauss LBBB criteria in Patients Diagnosed with the automated Glasgow LBBB criteria
}

Jakob Almer ${ }^{1}$; Robbert Zusterzeel, $\mathrm{MD}^{2}$; David G. Strauss, MD, PhD²; Elin Trägårdh, MD, $\mathrm{PhD}^{1}$; Charles Maynard, $\mathrm{PhD}^{3}$; Galen S. Wagner, $\mathrm{MD}^{4}$; Henrik Engblom, MD, $\mathrm{PhD}^{1}$

1 Department of Clinical physiology and Nuclear medicine, Skåne University Hospital and Lund University, Lund, Sweden

2 Center for Devices and Radiological Health, U.S. Food and Drug Administration, Silver Spring, Maryland, USA

3 Department of Health Services, University of Washington, Seattle, Washington, USA 4 Duke Clinical Research Institute, Durham, North Carolina, USA

Corresponding author: Henrik Engblom

Department of Clinical Physiology and Nuclear Medicine

Skåne University Hospital, Lund

221 85, Lund, Sweden

Phone: +46 46173304

Fax: +46 151769

E-mail: henrik.engblom@med.lu.se

Word count: 2819 


\section{ABSTRACT (max 150 words)}

Introduction. About one-third of patients undergoing cardiac resynchronization therapy because of left bundle branch block (LBBB) and heart failure do not improve. Strauss et al. have developed strict criteria to more accurately define complete LBBB in this patient group. The aim of this study was to investigate the prevalence of the manual Strauss criteria for LBBB (QRS $\geq 140 \mathrm{~ms}$ in men, $\geq 130 \mathrm{~ms}$ in women, along with mid-QRS notching/slurring) in consecutive patients who have been diagnosed with LBBB by the automated Glasgow criteria (QRS $\geq 120 \mathrm{~ms}$ ). Method. In 158 consecutive patients (78 females) diagnosed with LBBB according to the Glasgow criteria, the Strauss criteria were applied. Results \& Conclusion. A majority of patients $(87 \%)$ diagnosed with LBBB using the Glasgow criteria were positive for the Strauss criteria. In 70\% (13/20) of the cases of disagreement the reason for disagreement was short QRS duration.

\section{Keywords}

ECG, LBBB, CRT, diagnostic criteria 


\section{INTRODUCTION}

Cardiac resynchronization therapy (CRT) is used to treat patients who have clinical evidence of heart failure, low ejection fraction (EF) and left bundle branch block (LBBB). However, according to emerging clinical evidence, CRT may only achieve benefit in patients in whom complete block of the left bundle branch is truly present (1-5). In the presence of complete LBBB, the left ventricle (LV) myocardium is activated dyssynchronously because of the absence of impulse conduction through the interventricular His-Purkinje system of the left bundle branch. Thus the LV myocardium can only be electrically activated by trans-septal conduction from the right bundle branch and subsequent intramyocardial conduction from the septal to the lateral LV walls. This pathophysiology is in contrast to the conduction delay caused by slowed conduction through the LV myocardium in the presence of normal left bundle function $(6,7)$, which can also cause marked QRS complex prolongation, with or without LBBB pattern $(8,9)$.

Strauss et al. have recently developed new strict criteria to more accurately define complete LBBB in the setting of heart failure and CRT eligibility (referred to as the Strauss criteria throughout the paper)(10). These include the general evidence of LV conduction delay; QS or rS (in V1 and V2) and QRS duration $\geq 140 \mathrm{~ms}$ (males) or $\geq 130 \mathrm{~ms}$ (females), and specific evidence of LBBB with mid-QRS notch or slur in two or more of the following leads: V1, V2, V5, V6, I and aVL (Appendix 1). Recent studies have shown that consideration of Strauss criteria (2) and other strict LBBB criteria (11-13), sharing the QRS morphology criteria of the Strauss criteria, significantly improve prediction of CRT response and clinical outcome. Furthermore, Strauss criteria increase the specificity of complete LBBB diagnosis in the presence of LV hypertrophy/dilatation and incomplete LBBB (14). However, the applicability of Strauss criteria in a general consecutive LBBB population is not known.

One commercially available set of ECG criteria for LBBB, used to define a general 
LBBB population, was developed by Macfarlane and coworkers (15) at the Glasgow Royal Infirmary (GRI) and is used by over 20 ECG vendors worldwide. This computer algorithm is referred to as the Glasgow criteria throughout the paper (Appendix 2). The Glasgow criteria are in accordance with the QRS morphology criteria of the 1985 World Health Organization (WHO) and International Society and Federation for Cardiology (ISFC) taskforce criteria for LBBB (Appendix 3)(16), and the AHA/ACCF/HRS guidelines (17). The main difference between the Glasgow criteria and Strauss criteria is the difference in QRS thresholds, where the former use $\geq 120 \mathrm{~ms}$ in any two leads in comparison to $\geq 140 \mathrm{~ms}$ (males) or $\geq 130 \mathrm{~ms}$ (females) with the latter.

The aims of this study were 1) to investigate the prevalence of manual Strauss criteria, in a general LBBB population diagnosed with the automated Glasgow LBBB criteria, and 2) to determine the reason for discrepancies in diagnosing LBBB in these patients.

\section{METHODS}

\section{Study population}

Patients who received a resting ECG examination at the Department of Clinical Physiology, Skåne University Hospital, Lund between July 182012 and March 242014 were considered for inclusion in the study. Specific criteria for inclusion were patients over 18 years of age who had been diagnosed with LBBB according to the Glasgow criteria incorporated into automated software at the department (GRI, Glasgow Royal Infirmary, version 26.3.4/1.5) (15) with manual review by an experienced observer (HE) with regards to poor signal quality and/or other artifacts making the ECG uninterpretable (Appendix 2). The cardiac functional status or CRT treatment eligibility of these patients was not known or recorded for the present study. Thus, the patient population was not selected based on severe congestive heart failure and being candidates for CRT, but represents a general consecutive LBBB population. 
This study was approved by the regional ethics committee (Lund, Sweden), and all patients gave their informed consent to participate.

\section{ECG analysis}

Automated analysis based on Glasgow LBBB criteria.

The ECGs were recorded using an EC Sense system (Cardiolex, Sweden) and automatically analyzed using GRI, version 26.3.4/1.5 (Appendix 2)(15). The ECGs were recorded at a sample rate of $2000 \mathrm{~Hz}$ and stored at $1000 \mathrm{~Hz}$. The Glasgow LBBB criteria include several ECG characteristics that within this study have been grouped as general or specific criteria:

- General criteria

$\circ$ a) QRS duration $\geq 120$ in any two leads

○) QS or rS morphology with prolonged Q or S duration in V1 and V2

- Specific criteria

$\circ$ c) QRS slope in the later half of the QRS complex is decreased

$\circ$ d) Abnormal R wave morphology (in I, V5 and V6)

The general criteria select ECGs with a conduction delay originating (a) from the ventricles and (b) specifically from the left ventricle. The specific criteria (c and d) aim at distinguishing LBBB from other ventricular conduction delays. A detailed description of the Glasgow computer algorithm for diagnosing LBBB is shown in Appendix 2 (15).

\section{Manual analysis of the Strauss LBBB criteria.}

Two observers blinded to all clinical data except for gender manually analyzed the ECGs with regards to the new proposed criteria by Strauss et al. One experienced observer (RZ) and one control observer (JA) were included. In case of disagreement in the analysis, the observers attempted to resolve the disagreement, and if that was not possible an additional 
expert observer (GW) was consulted.

The criteria by Strauss et al. were, like the Glasgow criteria, divided into general and specific criteria. The criteria include (Appendix 1):

- General

- a) Global QRS duration $\geq 140 \mathrm{~ms}$ (males) or $\geq 130 \mathrm{~ms}$ (females)

○ b) QS or rS in V1 and V2

- Specific

- c) Mid-QRS notch or slur in two or more of the following leads: V1, V2, V5, V6, I and aVL

Again, the general criteria select ECGs with a conduction delay originating (a) from the ventricles and (b) specifically from the left ventricle. The specific criterion (c) aims at distinguishing LBBB from other left ventricular conduction delays. The global QRS duration as part of the Strauss criteria was defined as the time from earliest onset of the QRS complex, in any lead, to the latest offset of the QRS complex, in any lead. These measurements were made by the automated software. Manual over-read was conducted through digital viewing of the ECGs by reviewing of the position of the computer generated fiducials indicating QRS starting- and endpoint. When fiducials were incorrectly positioned manual measurement of the QRS duration was performed. The other subcriteria, (b) and (c), were manually recorded from median complex ECGs at $50 \mathrm{~mm} / \mathrm{s}$ by the two observers.

Within this study, mid-QRS was defined as approximately the mid half (50\%) of the QRS duration i.e. from $25 \%$ to $75 \%$ duration (the second and third quarter; Figure 1A). Notching in the QRS complex was defined as a sudden change, within a slope (waveform), in direction $\geq 90^{\circ}$ (Figure 1B) (18). Slurring was defined as a sudden change in the slope of a waveform with change in direction $0-90^{\circ}(<0.05 \mathrm{mV}$; Figure 1C). If mid-QRS notching or slurring existed the affected leads were recorded as well as the timing of the notch or slur. 


\section{Statistical analysis}

To test if the number of patients positive for Strauss criteria was significantly different from the patients diagnosed with LBBB using the Glasgow criteria, a 95\% confidence interval for the proportion of patients positive for the Strauss criteria was calculated. Second, the association between patient characteristics and meeting Strauss criteria was examined with the chi-square statistic for categorical variables and the two sample t-test for continuous variables. A p value of $<0.05$ was considered to indicate statistical significance. SPSS version 19.0 was used for data analysis.

\section{RESULTS}

The study population consisted of 158 patients ( 78 females), all positive for the Glasgow LBBB criteria. Patient characteristics are shown in Table 1 and differences between the groups that met the Strauss criteria and those who did not are shown in Table 2.

One-hundred thirty-eight out of the 158 patients (87\% [95\% confidence interval 81\%92\%]) were positive for the Strauss criteria (Table 1). Figure 2 shows the different reasons for not meeting Strauss criteria in those 20 patients, of whom $50 \%$ were females. Out of the 158 patients, 144 (91\%) were positive for subcriterion (a) of the Strauss criteria (QRS duration $\geq 140 \mathrm{~ms}$ [males] or $\geq 130 \mathrm{~ms}$ [females]), 158 (100\%) were positive for subcriterion (b) (QS or rS in V1 and V2) and 151 (96\%) were positive for subcriterion (c) (mid-QRS notch or slur in two or more of the following leads V1, V2, V5, V6, I and aVL) (Table 1). All patients had a mid-QRS notch or slur in one or more leads. The mean timing of the mid-QRS notch or slur was $52 \pm 11 \mathrm{~ms}$.

Figure 3A shows an example of an ECG with LBBB according to the Glasgow criteria where subcriteria (a) and (c) of the Strauss criteria were not met. Figure 3B shows an example ECG of a female patient not meeting Strauss LBBB criteria because of not meeting 
subcriterion (a).

Interobserver agreement on whether patients met Strauss criteria was 93\% (147/158). In all cases where observers did not agree initially (11/158), the disagreement was related to subcriterion (c). In 6 of those cases a third expert observer (GW) was consulted to reach a consensus.

\section{DISCUSSION}

The prevalence of the LBBB criteria defined by Strauss et al. in a group of patients that have been diagnosed with LBBB according the Glasgow LBBB criteria was $87 \%$. The reason for not meeting Strauss criteria was mainly due to short QRS duration $(<130 \mathrm{~ms}$ and $<140 \mathrm{~ms}$ for females and males, respectively).

The findings in the present study indicate that approximately $13 \%(20 / 158)$ of patients diagnosed with Glasgow LBBB criteria do not meet the Strauss criteria. The Glasgow criteria are in accordance with current AHA/ACCF/HRS guidelines regarding the QRS duration threshold ( $\geq 120 \mathrm{~ms})$ (17). The QRS thresholds ( $\geq 130 \mathrm{~ms}$ and $\geq 140 \mathrm{~ms}$ for females and males, respectively) proposed for the Strauss criteria are based on the electrical depolarization delay of at least $60 \mathrm{~ms}$, from normal heart mean QRS duration of $92.7 \pm 9.3$ ms (males) and $87.1 \pm 8.7 \mathrm{~ms}$ (females), that occurs during $\operatorname{LBBB}(10,19)$. Previous studies have shown that the benefit from CRT decreases with shorter QRS duration in patients diagnosed with $\operatorname{LBBB}(9,10)$. Furthermore, the suggested difference in QRS duration thresholds between females and males is supported by recent findings by Zusterzeel et al. showing a significant difference in benefit of CRT treatment between males and females with QRS duration between 130-149 ms (20).

Gettes et al. (21) suggest that the ECG diagnosis of LBBB should be standardized to that recommended by the WHO with retention of the minimum duration of $120 \mathrm{~ms}$ (current 
WHO criteria do not define a QRS duration threshold; Appendix 3). Furthermore, they point out that there is no intrinsic reason to require identical ECG criteria for LBBB and the selection criteria for patients with LBBB and heart failure who are most likely to benefit from resynchronization therapy. This approach is intriguing and acknowledges the fact that the underlying pathophysiology is different in patients with severe heart failure and LBBB compared to patients with LBBB without significant influence on cardiac function. Thus, it might be that the Strauss criteria should be applied in heart failure patients eligible for CRT and not in a general LBBB population, such as the one included in the present study, to avoid false negative LBBB diagnosis due to failure of meeting the higher QRS duration threshold. Hence, patients with LBBB morphology in the present study who did not meet the Strauss criteria due to the short QRS duration ( $<140 \mathrm{~ms}$ for males and $<130 \mathrm{~ms}$ for females) likely had a LBBB. Possibly some of the patients in this group had small hearts explaining why the QRS duration threshold was not met. Therefore, it might be more accurate to use a shorter QRS duration threshold (e.g $120 \mathrm{~ms}$ ) in accordance with the Glasgow criteria and recommended by AHA/ACCF/HRS $(16,17,21)$ when diagnosing LBBB in a general population. However, this requires further study.

As expected, all patients met subcriterion (b) of the Strauss criteria (QS or rS in V1 and V2) given the similarities of QRS morphology criteria in V1 and V2 existing in both the Glasgow criteria and Strauss criteria.

In $96 \%$ (151/158) of patients diagnosed with LBBB according to the Glasgow criteria, subcriterion (c) (mid-QRS notch or slur in two of the pre-specified leads) of the Strauss criteria was met. This high level of agreement between the two sets of criteria speaks to their similarities in sensitivity for mid-QRS notches and slurs. This could be explained by how the Glasgow criteria regard QRS morphology features, such as decreased QRS slope in the later half of the QRS complex and R wave morphology (in I, V5 and V6), making it sensitive for 
notches and slurs. In the 7 patients negative for Strauss criteria due to not meeting subcriterion (c), all had mid-QRS notches or slurs, but not in the pre-specified leads $(n=1)$, or only in one of them $(n=6)$. It should be noted that mid QRS notching and/or slurring was recorded already in 1956 by Grant et al. (7) as a significant criteria for complete LBBB identification. Furthermore, mid QRS notching and/or slurring that exists in both the Strauss criteria and the Glasgow criteria, are also represented by corresponding subcriteria in the AHA/ACCF/HRS guidelines as well as the WHO criteria.

Previous studies have shown that about one-third of the patients diagnosed with LBBB have no benefit from CRT (3-5). One possible reason for unsuccessful CRT treatment would be that the patients have been falsely diagnosed with LBBB when slow conduction is caused by other myocardial disease than LBBB (8). There are, however, other reasons for patients lacking benefit from CRT treatment, such as presence of myocardial fibrosis or other structural changes that coexist with the LBBB. Furthermore, optimization of the CRT treatment itself, regardless of underlying myocardial disease, is important as highlighted by van Deursen et al. (11).

In the majority of cases there was agreement between the two observers (93\%; 147/158). All the cases of disagreement were related to subcriterion (c) (presence of notch/slur), regarding in which leads notches and slurs were present. Thus, future studies should evaluate computer algorithms for detecting mid-QRS notches or slurs, as previously done for late QRS notches and slurs by Clark et al. (18) to decrease inter-observer variability.

\section{Limitations}

The findings in the present study should be interpreted in the light of some limitations. First, the definition of mid-QRS in the present study was arbitrary, since there is no standard definition of this term. The mean timing recorded by the observers for mid-QRS notch or slur 
was $52 \mathrm{~ms}$, where a definition of $25 \%-75 \%$ of the QRS duration was used for mid-QRS (Figure 1A). Secondly, this study was undertaken in a relatively small sample size using only one commercially available LBBB algorithm as baseline; the Glasgow criteria (version 26.3.4/1.5 of GRI). The rationale for only using one set of commercially available criteria is that the Glasgow criteria are applied at the Department of Clinical Physiology, Skåne University Hospital where the study was conducted. However, since the Glasgow criteria to a large extent are in accordance with the recommendations from AHA/ACCF/HRS, the findings in the present study may have a more general impact on the diagnosis of LBBB. Larger clinical studies also including other commercially available software packages for automated ECG analysis are still warranted for further development of diagnostic LBBB criteria in different clinical situations. Thirdly, there was no measure of myocardial contractility obtained in the present study. This would have enabled evaluation of presence and amount of dyssynchrony in the patients that met the Strauss criteria in comparison to those who did not, and thereby would have provided an estimate of the clinical significance of the findings.

\section{Conclusion}

The majority of patients (87\%) diagnosed with LBBB using the Glasgow criteria in this study were positive for the LBBB criteria developed by Strauss et al. The primary reason for disagreement was related to differences in QRS duration thresholds. Further study is warranted to determine the most appropriate criteria in a general LBBB population in contrast to a selected patient group eligible for CRT treatment.

\section{Acknowledgement}

We wish to acknowledge the technical expertise and ECG processing provided by Britt-Marie 
Gunnarsson (engineer, Skane University Hospital Lund). The study has been supported by the Swedish Heart and Lung foundation, Region of Scania and the Medical Faculty of Lund University. This study was supported by a research fellowship from the Oak Ridge Institute of Science and Education through an interagency agreement between the U.S. Department of Energy and the FDA.

\section{Disclaimer}

The mention of commercial products, their sources, or their use in connection with material reported herein is not to be construed as either an actual or implied endorsement of such products by the Department of Health and Human Services. 


\section{REFERENCES}

(1) Bristow MR, Saxon LA, Boehmer J, Kreuger S, Kass DA, De Marco T. Cardiacresynchronization therapy with or without an implantable defibrillator in advanced chronic heart failure. N Engl J Med 2004;350(21):2140-50.

(2) Risum N, Strauss DG, Sogaard P, Loring Z, Hansen TF, Bruun NE, et al. Left bundlebranch block: The relationship between electrocardiogram electrical activation and echocardiography mechanical contraction. American Heart Journal 2013;166(2):340-48.

(3) Zareba W, Klein H, Cygankiewicz I, Hall WJ, Goldberger J, Daubert JP, et al. CRT-D effectiveness by QRS duration and morphology in the MADIT-CRT patients. Heart Rhythm 2010;7(5):S25.

(4) Moss AJ, Hall WJ, Cannom DS, Klein H, Brown MW, Daubert JP, et al. CardiacResynchronization Therapy for the Prevention of Heart-Failure Events. $N$ Engl J Med 2009;361(14):1329-38.

(5) Bilchick KC, Kamath S, DiMarco JP, Stukenborg GJ. Bundle-branch block morphology and other predictors of outcome after cardiac resynchronization therapy in Medicare patients. Circulation 2010;122(20):2022-30.

(6) Vassallo JA, Cassidy DM, Marchlinski FE, Buxton AE, Waxman HL, Doherty JU, et al. Endocardial activation of left bundle branch block. Circulation 1984;69(5):914-23.

(7) Grant RP, Dodge HT. Mechanisms of QRS complex prolongation in man; left ventricular 
conduction disturbances. Am J Med 1956;20(6):834-52.

(8) Bacharova L, Szathmary V, Mateasik A. Electrocardiographic patterns of left bundlebranch block caused by intraventricular conduction impairment in working myocardium: a model study. Journal of Electrocardiology 2011;44(6):768-78.

(9) Strauss DG, Selvester RH. The QRS complex--a biomarker that "images" the heart: QRS scores to quantify myocardial scar in the presence of normal and abnormal ventricular conduction. Journal of Electrocardiology 2009;42(1):96.

(10) Strauss DG, Selvester RH, Wagner GS. Defining left bundle branch block in the era of cardiac resynchronization therapy. Am J Cardiol 2011;107(6):927-34.

(11) van Deursen CJ, Blaauw Y, Witjens MI, Debie L, Wecke L, Crijns HJ, et al. The value of the 12-lead ECG for evaluation and optimization of cardiac resynchronization therapy in daily clinical practice. Journal of Electrocardiology 2014;47(2):202-11.

(12) Tian Y, Zhang P, Li X, Gao Y, Zhu T, Wang L, et al. True complete left bundle branch block morphology strongly predicts good response to cardiac resynchronization therapy. Europace 2013;15(10):1499-1506.

(13) Mascioli G, Padeletti L, Sassone B, Zecchin M, Lucca E, Sacchi S, et al. Electrocardiographic Criteria of True Left Bundle Branch Block: A Simple Sign to Predict a Better Clinical and Instrumental Response to CRT. Pacing Clin Electrophysiol 2012;35(8):927-34. 
(14) Galeotti L, van Dam PM, Loring Z, Chan D, Strauss DG. Evaluating strict and conventional left bundle branch block criteria using electrocardiographic simulations. Europace 2013;15(12):1816-21.

(15) Macfarlane PW, Devine B, Clark E. The University of Glasgow (Uni-G) ECG analysis program. Comput Cardiol 2005(32):4.

(16) Willems JL, Robles de Medina EO, Bernard R, Coumel P, Fisch C, Krikler D, et al. Criteria for intraventricular conduction disturbances and pre-excitation. World Health Organizational/International Society and Federation for Cardiology Task Force Ad Hoc. J Am Coll Cardiol 1985;5(6):1261-75.

(17) Surawicz B, Childers R, Deal BJ, Gettes LS, Bailey JJ, Gorgels A, et al. AHA/ACCF/HRS recommendations for the standardization and interpretation of the electrocardiogram: part III: intraventricular conduction disturbances: a scientific statement from the American Heart Association Electrocardiography and Arrhythmias Committee, Council on Clinical Cardiology; the American College of Cardiology Foundation; and the Heart Rhythm Society: endorsed by the International Society for Computerized Electrocardiology. Circulation 2009;119(10):235.

(18) Clark EN, Katibi I, Macfarlane PW. Automatic detection of end QRS notching or slurring. Journal of Electrocardiology 2014;47(2):151-4.

(19) Macfarlane PW, Lawrie T. Appendix 1: Normal limits. Comprehensive 
Electrocardiology: Theory and Practice in Health and Disease New York: Pergamon Press; 1989. p. 1441-1526.

(20) Zusterzeel R, Selzman KA, Sanders WE, Caños DA, O'Callaghan KM, Carpenter JL, et al. Cardiac Resynchronization Therapy in Women: US Food and Drug Administration metaanalysis of patient-level data. JAMA Internal Medicine 2014;174(8):1340-8.

(21) Gettes LS, Kligfield P. Should electrocardiogram criteria for the diagnosis of left bundle-branch block be revised? Journal of Electrocardiology 2012;45(5):500-4. 


\section{TABLES}

Table 1: Population characteristics

\begin{tabular}{lc}
\hline & All patients (N=158) \\
\hline Gender (males) & $80(51 \%)$ \\
Mean age (years) & $69 \pm 12^{\mathrm{a}}$ \\
Mean QRS duration (ms) & $150 \pm 16^{\mathrm{a}}$ \\
Positive for Strauss LBBB criteria & $138(87 \%)$ \\
Positive for subcriterion a of Strauss LBBB criteria. ${ }^{\mathrm{b}}$ & $144(91 \%)$ \\
Positive for subcriterion b of Strauss LBBB criteria. ${ }^{\mathrm{b}}$ & $158(100 \%)$ \\
Positive for subcriterion c of Strauss LBBB criteria. ${ }^{\mathrm{b}}$ & $150(95 \%)$ \\
Any notch or slur & $158(100 \%)$ \\
Mid-QRS notch or slur & $158(100 \%)$ \\
Mean timing of mid-QRS notch or slur (ms) & $52 \pm 11$ \\
\hline
\end{tabular}

a) Mean \pm standard deviation

b) See Appendix A

Table 2: Baseline characteristics according to Strauss LBBB criteria

\begin{tabular}{lccc}
\hline & \multicolumn{2}{c}{ Strauss LBBB criteria } & P \\
\hline & $\begin{array}{c}\text { Positive } \\
(\mathrm{n}=138)\end{array}$ & $\begin{array}{c}\text { Negative } \\
(\mathrm{n}=20)\end{array}$ & \\
Males & $70(51 \%)$ & $10(50 \%)$ & $0.95^{\mathrm{b}}$ \\
Age (years) & $69 \pm 11^{\mathrm{a}}$ & $70 \pm 14^{\mathrm{a}}$ & $0.71^{\mathrm{c}}$ \\
Mean QRS & $153 \pm 14^{\mathrm{a}}$ & $135 \pm 13^{\mathrm{a}}$ & $<0.0001^{\mathrm{c}}$ \\
duration (ms) & & \\
\hline a) $\quad$ Mean \pm standard deviation & & \\
b) Calculated from chi-square test & & \\
c) Calculated from two sample t-test &
\end{tabular}




\section{FIGURE LEGENDS}

\section{Figure 1}

Definition of mid-QRS notches and slurs in the present study. A) Mid-QRS was defined as the half $(50 \%)$ of the QRS duration i.e. from $25 \%$ to $75 \%$ duration (the second and third quarter). B) Notching in the QRS complex was defined as a sudden change, within a slope (waveform), in direction $\geq 90^{\circ} .{ }^{12}$ C) Slurring was defined as a sudden change in the slope of a waveform with change in direction $0-90^{\circ}(<0.05 \mathrm{mV})$.

\section{Figure 2}

Distribution of patients not meeting the Strauss LBBB criteria based on subcriteria. Subcriteria (b) was met for all patients.

\section{Figure 3}

Example ECGs. (A) Example of a male patient not meeting the Strauss LBBB criteria due to not meeting subcriteria (a) and (c), QRS duration <140 ms (130ms) and no mid-QRS notch or slur in two of the following leads V1, V2, V5, V6, aVL and I. (B) Example of a female patient not meeting Strauss LBBB criteria because of not meeting subcriterion (a), QRS duration $<130(124 \mathrm{~ms})$ but where observers deem that the ECG could be a true LBBB morphologically. 
FIGURES

Figure 1

A.

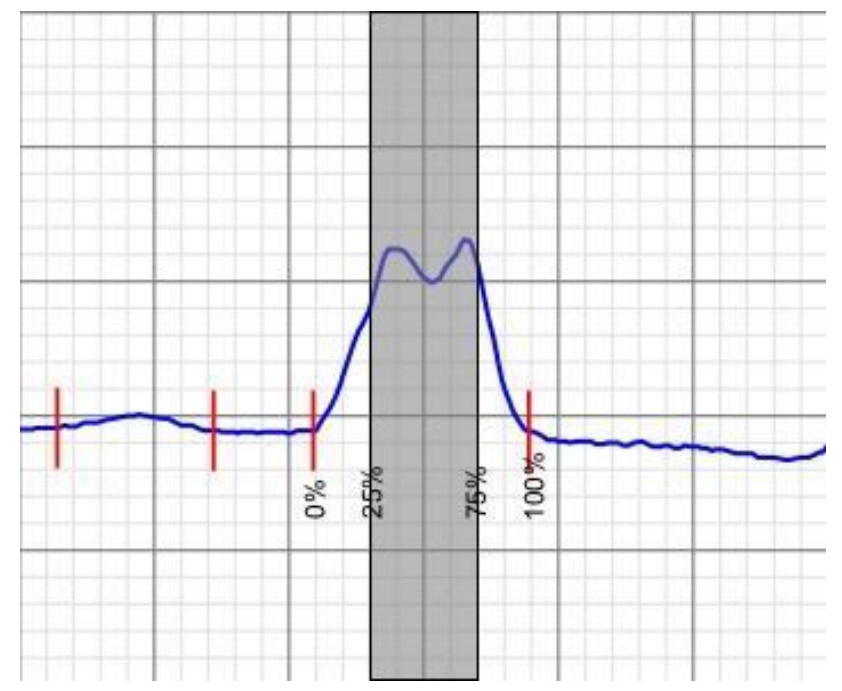

B.

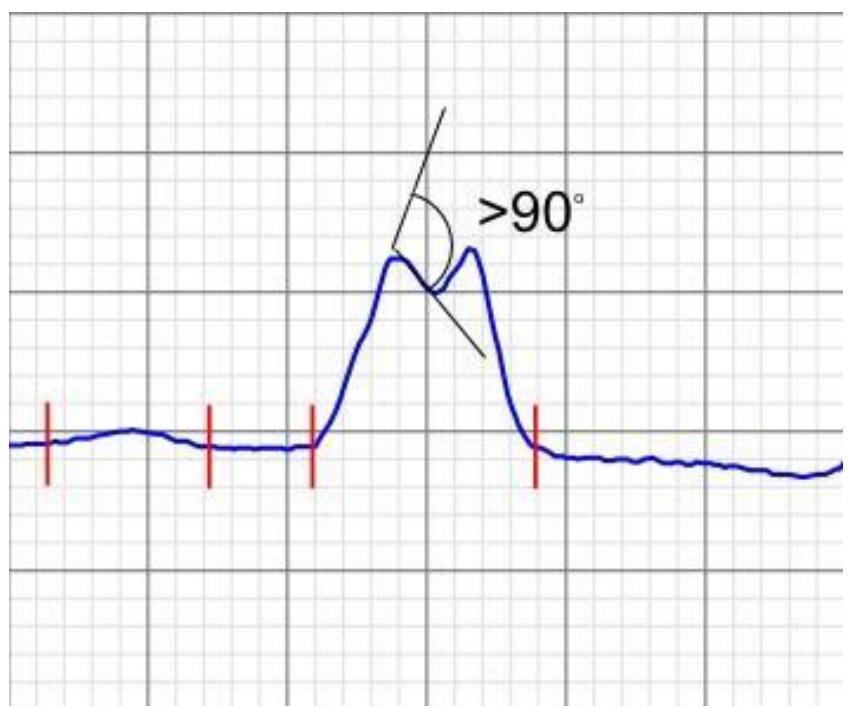


C.

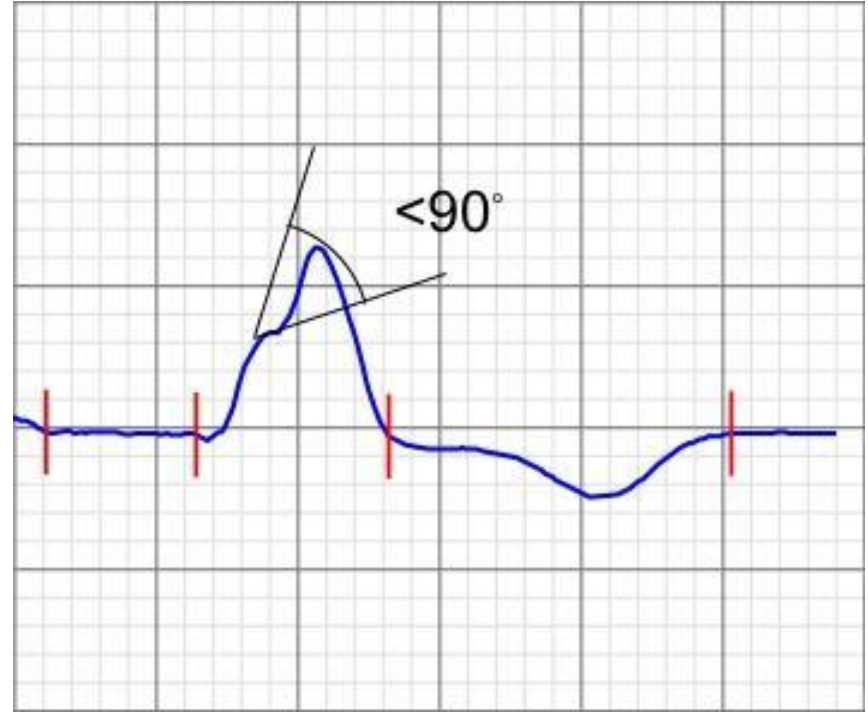


Figure 2

\section{Reasons for not meeting Strauss criteria based on subcriteria}

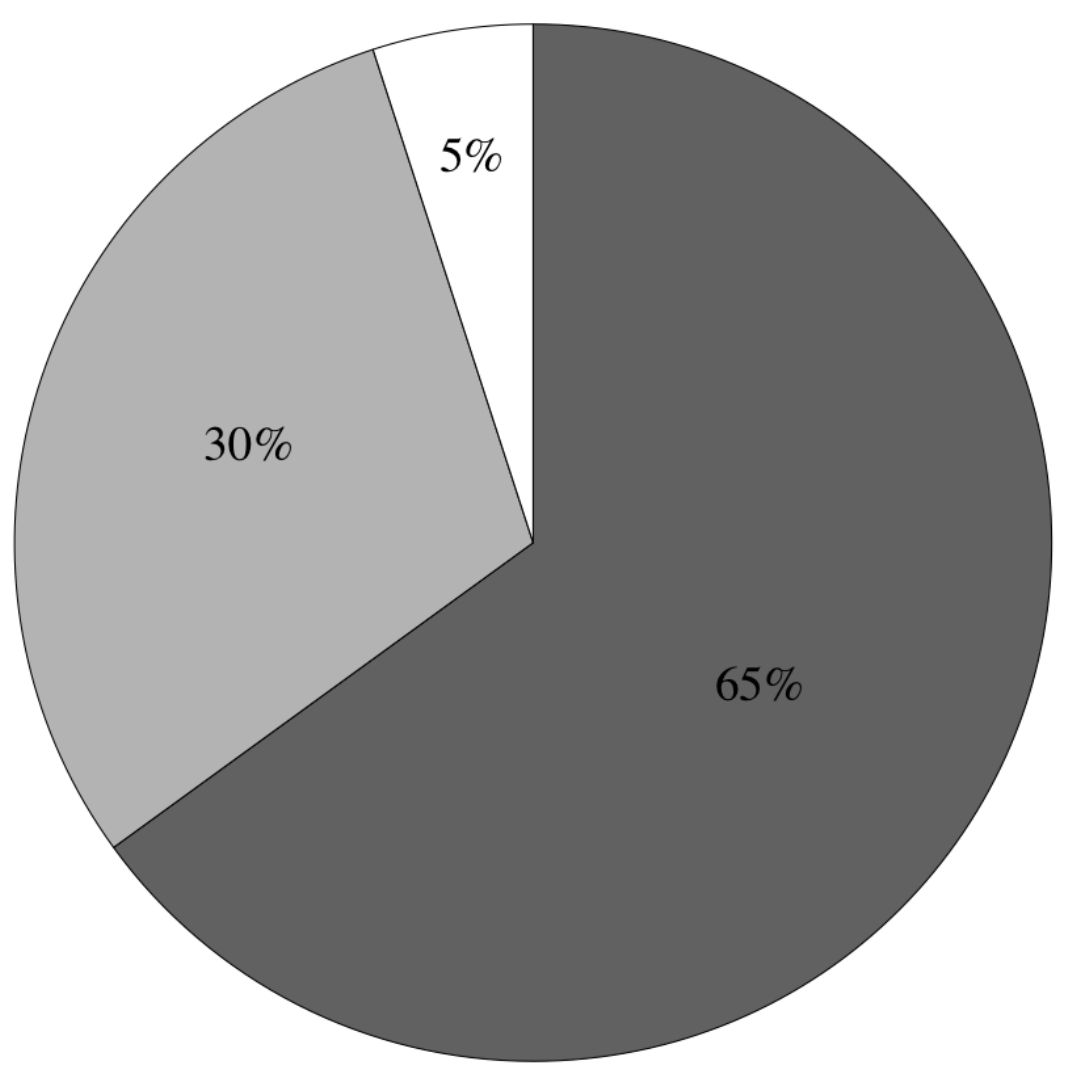

$\square$ Subcriteria (a); Absolute QRS duration $\geq 140 \mathrm{~ms}$ for men $\geq 130$ for women.

$\square$ Subcriteria (c); Mid-QRS notch or slur in $\geq 2$ of the following leads: V1, V2, V5, V6, I and aVL.

$\square$ Subcriteria (a) and (c) 
Figure 3

A.

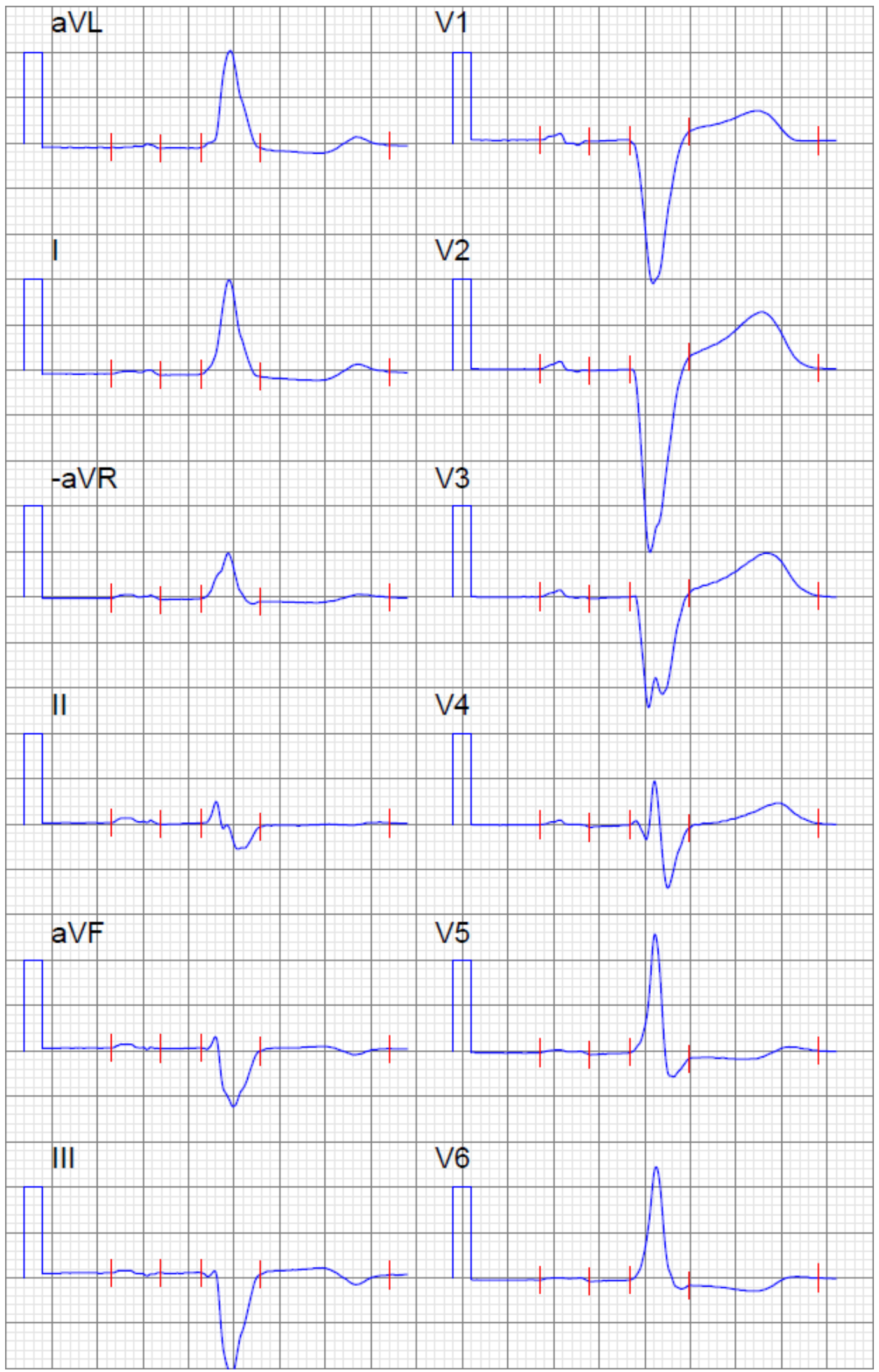


B.

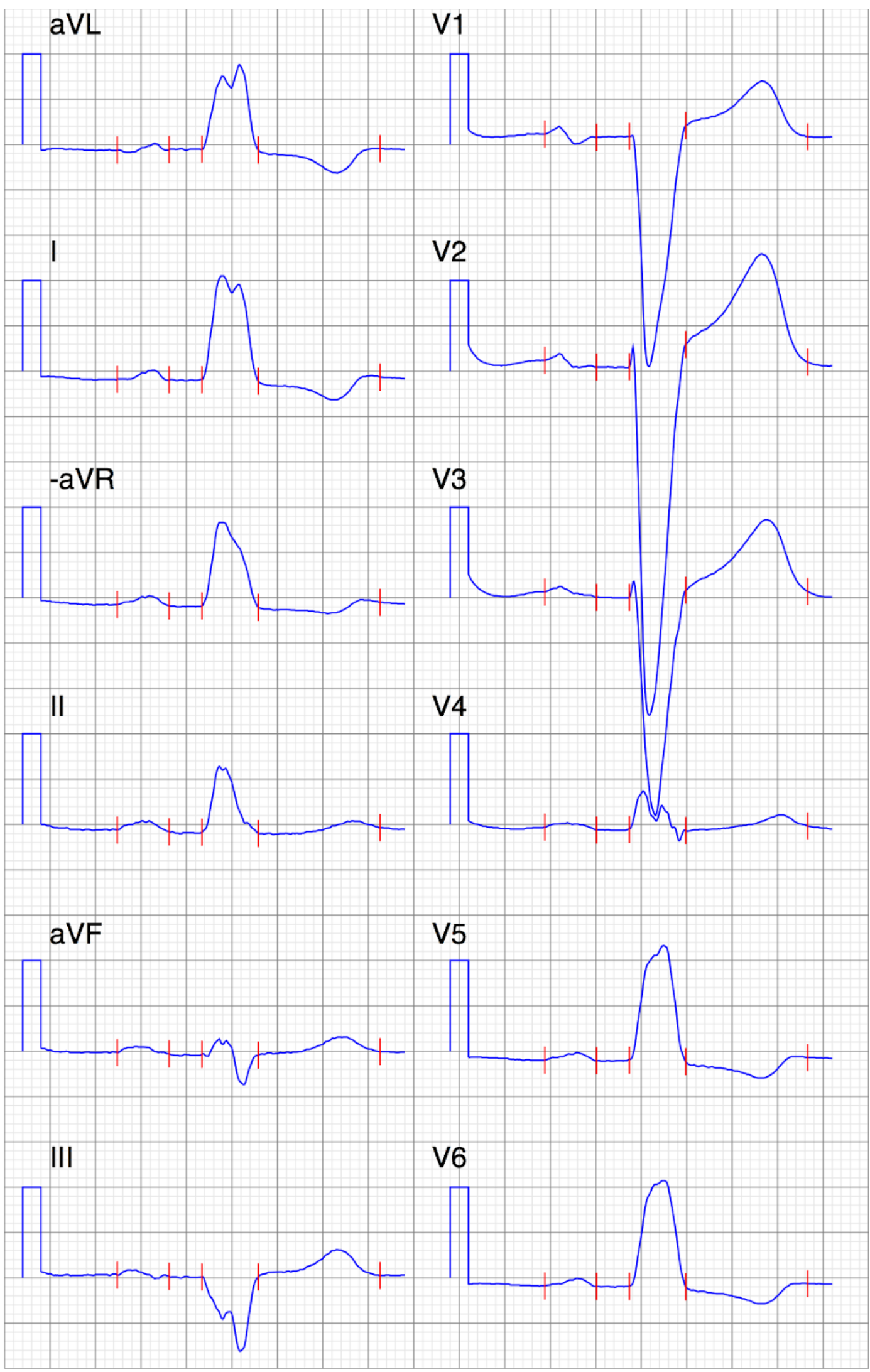




\section{APPENDICIES}

\section{LBBB criteria according to Strauss et al.(10)}

(a)

i. Absolute QRS duration $\geq 140 \mathrm{~ms}$ (males).

or

ii. Absolute QRS duration $\geq 130 \mathrm{~ms}$ (females).

and (b) QS or rS in V1 and V2.

and (c) Mid QRS notching or slurring in $\geq 2$ of leads V1, V2, V5, V6, I, and aVL.

\section{Criteria for Left bundle branch block by version 26.3.4/1.5 of GRI (Glasgow Royal}

Infirmary, Macfarlane et al)(15)

A.

(a) the QRS spatial velocities at any two of $4 / 8,5 / 8$ and $6 / 8<100 \mathrm{mV} / \mathrm{sec}$

and (b) i. in Lead I, V5 or V6:R > 100, with $\mathrm{Q}>-0.02 \mathrm{mV}$

or ii. in Lead I, V5 or V6: $\mathrm{R}^{\prime}>100$, with $\mathrm{S}>-0.02 \mathrm{mV}$

and (c) in V1, either Q or S $\geq 90$ with amplitude $<-1 \mathrm{mV}$

and $\quad(\mathrm{d}) \quad\left(\mathrm{R}+\mathrm{R}^{\prime}\right)$ duration summed over I, V5 and V6 $>270$

and (e) $\quad \mathrm{R}$ amplitude/R duration $<20$ in I and (V5 or V6) with $|\mathrm{R} / \mathrm{S}|>4$

and (f) QRS duration $\geq 120$ in any two leads QRS duration

and $\quad(\mathrm{g}) \quad$ in $\mathrm{V} 2$, sum of $\mathrm{R}+\mathrm{R}^{\prime}<0.3 \mathrm{mV}$

or $\mathrm{B}$.

None of the previous statements is true and from the following criteria either: ( $a$ and $b$ and $c$ and $d$ and $f$ )

or (b and $\mathrm{d}$ and $\mathrm{e}$ and $\mathrm{f})$ is true 
(a) QRS duration > 120 in any two leads QRS duration

(b) i. in Lead I, V5 or V6: R > 100, with Q $>-0.02 \mathrm{mV}$

or ii. in Lead I, V5 or V6: $\mathrm{R}^{\prime}>100$, with $\mathrm{S}>-0.02 \mathrm{mV}$

(c) i. in Lead I, $\mathrm{S} \leq 35$, or $\mathrm{S} \geq-0.15 \mathrm{mV}$, or $|\mathrm{R} / \mathrm{S}| \geq 4$

and ii. in Lead $I, S^{\prime} \leq 35$, or $S^{\prime} \geq-0.15 m V$, or $\left|R^{\prime} / S^{\prime}\right| \geq 4$

(d) in V1 or V2, either Q or S $>100$, with corresponding amplitude $<-1.0 \mathrm{mV}$

(e) the QRS spatial velocity at $4 / 8$ and $5 / 8<100 \mathrm{mV} / \mathrm{sec}$

(f) $\quad\left(\mathrm{R}+\mathrm{R}^{\prime}\right)$ duration summed over I, V5 and V6 $>270$

\section{1985 World Health Organization (WHO) and International Society and Federation} for Cardiology (ISFC) taskforce criteria for LBBB (16)

(a) QRS complex duration of 120 milliseconds or more

and (b) Broad, notched, or slurred R waves in leads I, aVL, V5 and V6 with occasional RS in leads V5 and/or V6

and (c) Absent Q in leads 1, V5, and V6

and (d) Prolongation of the time to the peak of the R wave to more than $60 \mathrm{~ms}$ in leads V5 and V6. 